\title{
On stabilized finite element methods based on the Scott-Zhang projector. Circumventing the inf-sup condition for the Stokes problem
}

\author{
Santiago Badia* \\ Centre Internacional de Mètodes Numèrics en Enginyeria (CIMNE), Parc Mediterrani de la Tecnologia, Esteve Terrades 5, 08860 Castelldefels, Spain \\ Universitat Politecnica de Catalunya, Jordi Girona 1-3, Edifici C1, 08034 Barcelona, Spain
}

\section{A R T I C L E I N F O}

\section{Article history:}

Received 14 May 2012

Received in revised form 16 July 2012

Accepted 31 July 2012

Available online 16 August 2012

\section{Keywords:}

Stabilized finite elements

Stokes problem

Indefinite systems

\begin{abstract}
A B S T R A C T
In this work we propose a stabilized finite element method that permits us to circumvent discrete infsup conditions, e.g. allowing equal order interpolation. The type of method we propose belongs to the family of symmetric stabilization techniques, which are based on the introduction of additional terms that penalize the difference between some quantities, i.e. the pressure gradient in the Stokes problem, and their finite element projections. The key feature of the formulation we propose is the definition of the projection to be used, a non-standard Scott-Zhang projector that is well-defined for $L^{1}(\Omega)$ functions. The resulting method has some appealing features: the projector is local and nested meshes or enriched spaces are not required.
\end{abstract}

(c) 2012 Elsevier B.V. All rights reserved.

\section{Introduction}

Many physical problems in science and engineering are modeled with partial differential equations without a coercivity property, e.g. the (Navier-)Stokes equations for incompressible flows, Darcy's problem for flux in porous media, and some versions of the Maxwell equations. At the continuous level, these saddle-point problems are well posed by virtue of some inf-sup condition. As model problem, let us consider Stokes' system on a bounded open domain $\Omega$ with homogeneous boundary conditions:

$-v \Delta u+\nabla p=f, \quad \nabla \cdot u=0,\left.\quad u\right|_{\partial \Omega}=0$,

where $u$ is the velocity, $p$ the pressure, $v$ the fluid viscosity and $f$ the body force. Using standard notation, we can state the problem in weak form: find $(u, p) \in H_{0}^{1}(\Omega) \times L_{0}^{2}(\Omega)$ such that

$c(u, p, v, q):=v(\nabla u, \nabla v)-(p, \nabla \cdot v)+(q, \nabla \cdot u)=\langle f, v\rangle=: \ell(v)$

for any $(v, q) \in H_{0}^{1}(\Omega) \times L_{0}^{2}(\Omega)$. For the Stokes problem, pressure stability relies on the following inf-sup condition: for any $p \in L_{0}^{2}(\Omega)$, there exists a $v_{p} \in H_{0}^{1}(\Omega)$ with unit norm such that $\beta\|p\| \geqslant\left(p, \nabla \cdot v_{p}\right)$, for some $\beta>0$. Unlike coercivity, inf-sup conditions are not inherited by sub-spaces of functions, complicating Galerkin approximations. We have to explicitly build finite element (FE) spaces that satisfy discrete versions of the inf-sup conditions, and appealing choices such as equal-order interpolation for all the

\footnotetext{
* Address: Universitat Politecnica de Catalunya, Jordi Girona 1-3, Edifici C1, 08034 Barcelona, Spain.

E-mail address: sbadia@cimne.upc.edu
}

unknowns cannot be used [8]. The situation is slightly more involved in general, since the well-posedness of saddle-point problems only requires coercivity on the kernel of the constraint operator, e.g. the Maxwell and Darcy problems [14].

The use of inf-sup stable formulations can be particularly impractical in multi-physics simulations of saddle-point systems, i.e. inductionless magnetohydrodynamics (MHD) (coupling Stokes and Darcy-type problems, see [21]) or incompressible visco-resistive MHD (coupling Stokes and Maxwell-type problems, see [3]). This approach requires different FE spaces for the different unknowns [22], complicating the integration subroutines and the matrix graph (a different graph for every block of the full matrix is needed). In other situations, in which different asymptotic limits of the problem (in terms of the physical parameters) lead to different saddle-point systems, e.g. the Brinkman model for creeping flow in porous media, the inf-sup stable approximation cannot lead in general to unconditional stability, since different limits require different FE spaces (see [1]).

Alternatively, we can consider stabilized FE techniques, which consist in the introduction of additional (stabilization) terms that provide the numerical method with the proper stability without the need to satisfy discrete inf-sup conditions. Initially, the stabilization terms were based on the residual at FE interiors, as in the popular Galerkin/least-squares (GLS) method [16] and the improved Variational Multiscale (VMS) method [17] proposed by Hughes and co-workers. Let us consider a partition $\mathcal{T}_{h}$ of $\Omega$ into tetrahedra/hexahedra, denoted by $K$, and conforming FE spaces $V_{h} \times Q_{h} \subset H_{0}^{1}(\Omega) \times L_{0}^{2}(\Omega)$. The stabilized methods GLS $(\theta=-1)$ and VMS $(\theta=1)$ read as: 


$$
\begin{gathered}
c\left(u_{h}, v_{h}, p_{h}, q_{h}\right)+\sum_{K \in \mathcal{T}_{h}} \delta_{K}\left(-\Delta u_{h}+\nabla p_{h}, \theta \Delta v_{h}+\nabla q_{h}\right)_{K} \\
=\ell\left(v_{h}\right)+\sum_{K \in \mathcal{T}_{h}} \delta_{K}\left\langle f, \theta \Delta v_{h}+\nabla q_{h}\right\rangle_{K},
\end{gathered}
$$

where $\delta_{K}$ is a numerical parameter to be defined later. These methods allow equal-order interpolation, are consistent and exhibit optimal convergence rates. On the other side, they are usually criticized for giving unphysical pressure boundary layers [5], for the additional cost involved in the evaluation of higher order derivatives and the weak inconsistency for first order approximations [18], the fact that the forcing term is also affected by the stabilization and the hard extension to transient problems [13], usually carried out via expensive space-time FEs (see e.g. [24]). Probably, the main shortcoming of GLS, VMS and related residual-based formulations is manifested when dealing with multi-physics applications. These stabilized FE formulations include a large number of additional coupling terms, which fill blocks that are zero for the Galerkin method; see $[21,3]$ for MHD applications. Another important problem related to these methods is the fact that they destroy the skew-symmetric form of the off-diagonal blocks (stabilized gradient and divergence matrix) in the Navier-Stokes and MHD systems, making pressuresegregation (fractional step) methods to lose their unconditional stability (see [4]).

The introduction of symmetric stabilization techniques represented one step further in the improvement of FE stabilization techniques, since they solve all the problems commented above.

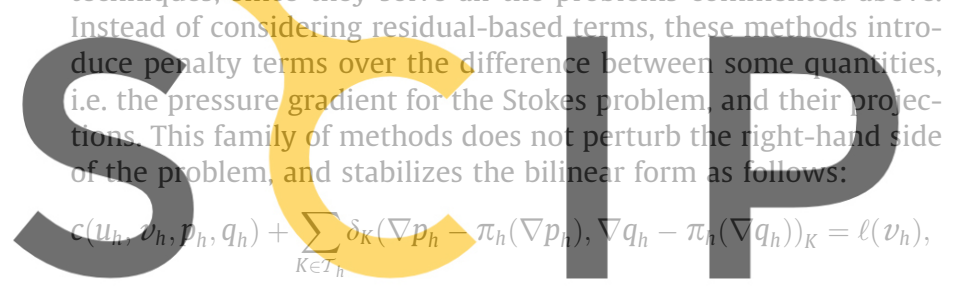

where $\pi_{h}(\cdot)$ is a FE projector; different definitions for $\pi_{h}(\cdot)$ lead to Regifstert fonnfmee ate hethips / / whawis cippedialycomisto tent, i.e. the stabilization term does not cancel for the exact solution but vanishes as the mesh size $h \searrow 0$ in such a way that optimal convergence is kept. Iviotivated by the inherited stability of fractional step methods, Codina and Blasco provided in their pioneering work [12] the first algorithm of this kind, based on the $L^{2}(\Omega)$-orthogonal projector, coined orthogonal subscales (OSS). Unfortunately, this projector is global, i.e. the stabilization term leads to a dense matrix. Certainly, the method is never computed this way, and the projection is usually sent to the right-hand side of the linear system. In case of solving transient problems, it can simply be treated explicitly. In those situations, for reasonably small time step sizes, the OSS method has perfect sense and it is an effective and simple algorithm, since the CPU cost per time step used for the computation of the global projections in negligible. On the contrary, to send the projection term to the right-hand side, and make it implicit via Richardson iterations (usually merged with nonlinear iterations [11]) is not advisable unless a very small time-step size is used, since it can drastically increase the number of nonlinear iterations or simply diverge; this approach is even harder to justify for linear problems as the Stokes system. An alternative consists in dealing with the exact matrix, explicitly solving the mass matrix systems for the projection evaluations at every iteration of an external Krylov solver. This approach is hard to implement and prevent us to use direct solvers, and by extension substructuring domain decomposition techniques with exact local solvers [25].

Becker and Braack envisaged in [5] an original way to avoid the global projections in [12]. Their method was later called local projection stabilization (LPS). The price to pay is a tighten requirement over the mesh partitions: specific hierarchical meshes were needed, since the method is based on the definition of fine and coarse FE spaces. On the other hand, the projection is not over the original FE space, as in [12], but on a discontinuous space of functions. The original LPS formulation has been lately denoted as two-level LPS, due to the requirement of two nested meshes for the definition of the stabilization terms. A one-level LPS formulation has also been designed [19], in which the fine space is attained with an enrichment of the coarse one via additional functions of bubble type. This way, we can eliminate the stringent mesh requirement but now a particular type of enriched FE spaces must be used.

The development of stabilized FE methods that allows one to circumvent inf-sup conditions has been almost entirely developed for the Stokes problem and the nonlinear Navier-Stokes equations. The extension to problems that only present coercivity in the kernel of the constraint operator is more recent. We refer to [2] for a detailed exposition of VMS and symmetric projection stabilization schemes for Darcy's and Maxwell's problems.

The purpose of this work is to present a new method, based on a particular $L^{1}(\Omega)$ Scott-Zhang projector that shares all the aforementioned benefits of symmetric stabilization techniques as well as: only local projections are required, no assumption over the mesh partition (e.g. nested meshes) is needed and no assumption over the FE spaces (e.g. equal-order Lagrangian FEs can be used without additional enrichment) is needed.

In Section 2, we introduce some notation, as well as the definition of the method and implementation aspects. Section 3 is devoted to the nunferical anatysis of the algprithm, both stability
and a priori error estimates. Son numerical tests are presented
in Section 4. Finally, we draw some conclusions in Section 5
2. Definition of the method
Let us conside the Stokes problem (2) for an open, bounded polyhedral domain $\Omega$ in $\mathbb{R}^{d}$, where $d=2,3$ is the space dimension. We will use standard notation for Sobolev spaces (see [7]). In pardiavantoadatseldersdonwivithoutethe (watersamerk $\omega \subset \Omega$, but the domain subscript is omitted for $\omega \equiv \Omega$ (analogously for the duality pairing $\langle\cdot, \cdot\rangle)$. The $L^{2}(\Omega)$ norm is denoted as $\|\cdot\|$. We will define the veiocity and pressure spaces as $V_{0} \equiv H_{0}^{1}(\Omega)$ and $Q \equiv L_{0}^{2}(\Omega)$, endowed with the norms $\|v\|_{V}:=v^{\frac{1}{2}}\|\nabla v\|$ and $\|q\|_{Q}:=v^{-\frac{1}{2}}\|q\|$, properly scaled with the fluid viscosity $v . C^{0}(\bar{\Omega})$ denotes the space of continuous functions. We will omit the $d$ superscript in vector-valued functional spaces.

Let us consider now a partition $\mathcal{T}_{h}$ of $\Omega$ into $d$-simplices, quadrilaterals $(d=2)$ or hexahedra $(d=3)$ where every $K \in \mathcal{T}_{h}$ is the image of a reference element $\widehat{K}$ through an affine mapping $F_{K}: \widehat{K} \rightarrow K$ (see [9, Chp. 2]); we can assume that every edge of $\widehat{K}$ has length one. $P_{r}(\widehat{K})$ is the space of complete polynomials of degree $r$ on $\widehat{K}$. For $d$-simplicial FE partitions, we define the space of element-wise discontinuous functions

$D_{h}:=\left\{v_{h}:\left.v_{h}\right|_{K} \circ F_{K} \in P_{r}(\widehat{K}), K \in \mathcal{T}_{h}\right\}$.

The continuous FE spaces are obtained by enforcing continuity, namely $V_{h}:=D_{h} \cap C^{0}(\bar{\Omega})$ and $Q_{h}:=D_{h} \cap C^{0}(\bar{\Omega}) \cap L_{0}^{2}(\Omega)$ for the velocity and pressure respectively. We will also make use of the FE space with null trace $V_{h, 0}:=V_{h} \cap H_{0}^{1}(\Omega)$. The order of approximation $r$ to be used for velocity and pressure approximations can be different. For quadrilaterals and hexahedra, the spaces are obtained by replacing $P_{r}(\widehat{K})$ by $Q_{r}(\widehat{K})$, the space of polynomials with maximum degree $r$ in each reference space coordinate on $\widehat{K}$.

For the FE space $V_{h}$ we denote by $\mathcal{N}_{h}$ the set of all interpolation nodes related to $\mathcal{T}_{h}$ and by $\left\{\phi^{a}\right\}_{a \in \mathcal{N}_{h}}$ the corresponding nodal basis of $V_{h}$. We also denote by $\mathcal{N}_{h}(K)$ the set of all nodes that belong to a FE $K$. Continuous FE functions can be written as $v_{h}=\sum_{a \in \mathcal{N}_{h}} v_{h}^{a} \phi^{a}(\mathbf{x})$, where $v_{h}^{a}$ denotes the nodal value of $v_{h}$ corresponding to $a$. Analo- 
gously, $\left\{\left.\phi^{a}(\mathbf{x})\right|_{K}\right\}_{K \in \mathcal{T}_{h}, a \in \mathcal{N}_{h}(K)}$ is the basis for $D_{h}$ and discontinuous FE functions are written as $v_{h}=\left.\left.\sum_{K \in \mathcal{T}_{h}} \sum_{a \in \mathcal{N}_{h}(K)} v_{h}\right|_{K} ^{a} \phi^{a}(\mathbf{x})\right|_{K}$, where $\left.v_{h}\right|_{K} ^{a}$ is the value of $v_{h}$ in the node $a$ at element $K$. Further, we denote the adjacency list $\mathcal{A}_{h}^{\text {nd }}(a)$ of a node $a \in \mathcal{N}_{h}$ as the set of nodes $c \in \mathcal{N}_{h}$ such that $a, c \in \mathcal{N}_{h}(K)$ for some $K \in \mathcal{T}_{h}$, i.e. the set of neighbor nodes. On the other hand, $\mathcal{A}_{h}^{\mathrm{el}}(a)$ is the set of elements $K \in \mathcal{T}_{h}$ such that $a \in \mathcal{N}_{h}(K)$, i.e. the set of neighbor elements.

\subsection{Nodal projection stabilization}

At this point, let us introduce a variant of the Scott-Zhang projector [23] which is well-defined for $L^{1}(\Omega)$ functions. The analysis in [23] does apply for this projector even though it is not the standard version, because it does not keep homogeneous boundary conditions (see Remark 1 ). We can build a node-to-element map $\kappa: \mathcal{N}_{h} \rightarrow \mathcal{T}_{h}$; for interior nodes there is only one choice, two choices for nodes on faces and possibly more for nodes on edges or vertices. Following [23], for any $a \in \mathcal{N}_{h}$ there is only one dual function $\psi^{a} \in P_{r}(K)$ (analogously for $Q_{r}(K)$ ) such that

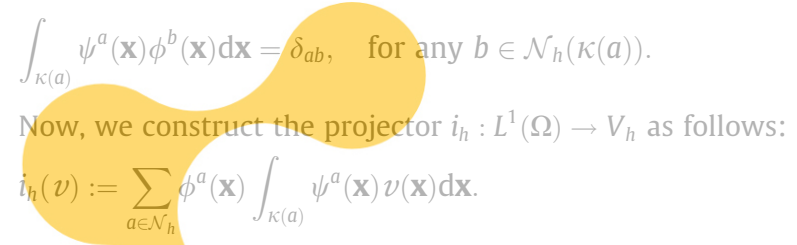

Let us also define $i_{h}^{\perp}(v):=v-i_{h}(v)$. With this notation, we are in
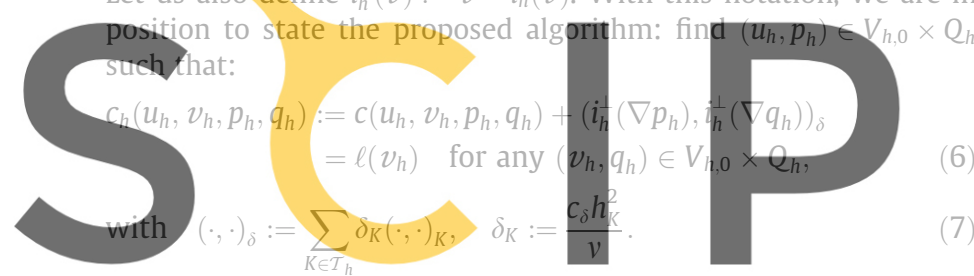

is the so-called stabilization parameter, $c_{\delta}$ being an

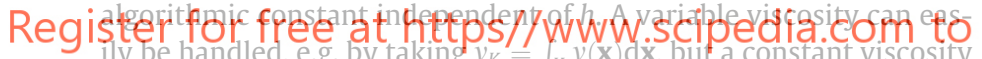
is assumed for the sake of clarity. We will refer to this method as nodal projection stabilization (NPS), since $i_{h}(\cdot)$ is a local projection defined node-wise. The method clearly belongs to the family of symmetric stabilization techniques.

Motivated by the stability analysis in the next section, we consider an alternative NPS scheme (6) by introducing a nodal definition of the stabilization parameter, denoted by $\delta_{a}$. Given a (generally discontinuous) FE element function $v_{h} \in D_{h}$, we define $\delta\left(v_{h}\right)(\mathbf{x}):=\left.\left.\sum_{K \in \mathcal{T}_{h}} \sum_{a \in \mathcal{N}_{h}} \delta_{a} v_{h}\right|_{K_{1}} ^{a} \phi\right|_{K} ^{a}(\mathbf{x}) \in D_{h}$. We define $\delta^{\frac{1}{2}}(\cdot)$ analogously, by replacing $\delta_{a}$ with $\delta_{a}^{2}$. Let us remark that $\delta(\cdot)$ keeps continuity and homogeneous boundary conditions, i.e. given $v_{h} \in V_{h}, \delta\left(v_{h}\right) \in V_{h}$ (idem for $V_{h, 0}$ ). The method consists in (6) with a different definition of the $\delta$-product:

$(\cdot, \cdot)_{\delta}:=\left(\delta^{\frac{1}{2}}(\cdot), \delta^{\frac{1}{2}}(\cdot)\right), \quad \delta_{a}:=\frac{c_{\delta} h_{a}^{2}}{v}, \quad$ with $h_{a}:=h_{\kappa(a)}$.

Alternative definitions of $h_{a}$ are possible (see Lemma 4). In this case, the stability analysis in Theorem 6 becomes slightly simpler.

This method is strongly related to the OSS formulation proposed by Codina and Blasco in [12], which uses instead the $L^{2}(\Omega)$ projector $\pi_{h}(v) \in V_{h}$, defined as the solution of

$\left(\pi_{h}(v), w_{h}\right)=\left(v, w_{h}\right), \quad$ for any $w_{h} \in V_{h}$.

However, this projector is not local, since it involves the solution of a global linear system with a Gramm matrix. On the other hand, LPS techniques share with NPS the fact that the projections are local, but the projectors are not defined over $V_{h}$ and they require additional assumptions over the mesh or FE spaces to be used. We refer to $[5,19]$ for a detailed definition of LPS techniques.
Remark 1. For the OSS formulation, it has been proposed to use the $L^{2}(\Omega)$ projector $\pi_{h, 0}$ onto $V_{h, 0}$ instead, i.e. the solution $\pi_{h, 0}(v) \in V_{h, 0}$ of

$\left(\pi_{h, 0}(v), w_{h}\right)=\left(v, w_{h}\right), \quad$ for any $w_{h} \in V_{h, 0}$.

It does not require Assumption 5 below to hold but can exhibits pressure boundary layers. Analogously for NPS, we can consider a projector $i_{h, 0}$ onto $V_{h, 0}$. It can be accomplished by modifying the projector (5) at nodes $a \in \partial \Omega$ only. For these boundary nodes, we can use the technique in [23], based on edge integrals, since $i_{h, 0}$ is only applied over $\nabla Q_{h}$ functions, which have well-defined traces on $\partial \Omega$. Alternatively, we can use the modification proposed by Girault and Lions [15], which simply consists in taking $i_{h}\left(\nabla q_{h}\right)^{a}$ equal to zero for boundary nodes. In fact, for the FE functions to be projected in method (6) these two techniques are equivalent.

\subsection{Implementation aspects}

In this section we will assume the equal-order interpolation case for the sake of clarity. Let us discuss how to implement the stabilization term in (6). The key ingredient is the computation of $i_{h}\left(\nabla p_{h}\right)$. By linearity we only need to consider the projection of the basis $\left\{\nabla \phi^{a}\right\}_{a \in N_{h}}$ of the discontinuous FE space $\nabla Q_{h}$. We have the following result.

Lemma 2. Let us assume that $r_{p} \leqslant r_{u}+1$, where $r_{u}$ and $r_{p}$ are the order of approximation for the velocity and pressure respectively.
Then, the matrix associated to the term $\left(i_{h}(\nabla \cdot), i_{h}(\nabla \cdot)\right): c_{h} \rightarrow Q_{h}^{\prime}$, can
be built as follows: for any $a b \in \mathcal{N}_{h}$
$\left(i_{h}\left(\nabla \phi^{a}\right), i_{h}\left(\nabla \phi^{b}\right)\right)=\sum_{c \in \mathcal{A}_{h}^{\text {nd }}(a) d} \sum_{\mathcal{A}_{h}^{\text {nd }}}\left(\left.\left.\nabla \phi^{a}\right|_{k(c)} ^{c} \cdot \nabla \phi^{b}\right|_{\kappa(d)} ^{d}\left(\phi^{c}, \phi^{d}\right)\right.$.
Proof. By the definition of the projector (5), we have: for any

$a \in \mathcal{N}_{h}$

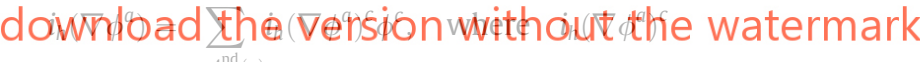$$
=\int_{\kappa(c)} \nabla \phi^{a}(\mathbf{x}) \psi^{c}(\mathbf{x}) \mathrm{d} \mathbf{x} .
$$

We have used the simple fact that $\left.\nabla \phi^{a}\right|_{\kappa(c)} ^{c}=0$ if $c \notin \mathcal{A}_{h}^{\text {nd }}(a)$, since $a \notin \kappa(c)$ and so $\left.\nabla \phi^{a}\right|_{\kappa(c)}=0$. Now, let us use the fact that, given a FE $K$, for any $q_{h} \in Q_{h}$ there exists a $v_{h} \in V_{h}$ such that $\left.\nabla q_{h}\right|_{K}=\left.v_{h}\right|_{K}$. This is true since $r_{p} \leqslant r_{u}+1$. Then, we can write $\left.\nabla \phi^{a}\right|_{K}=$ $\left.\left.\sum_{b \in \mathcal{N}_{h}(K)} \nabla \phi^{a}\right|_{K} ^{b} \phi^{b}\right|_{K}$. Using this result in (11) and the definition of the dual functions in (4) we readily get:

$$
\begin{aligned}
i_{h}\left(\nabla \phi^{a}\right)^{c} & =\left.\sum_{b \in \mathcal{N}_{h}(\kappa(c))} \int_{\kappa(c)} \nabla \phi^{a}\right|_{\kappa(c)} ^{b} \phi^{b}(\mathbf{x}) \psi^{c}(\mathbf{x}) \mathrm{d} \mathbf{x} \\
& =\left.\nabla \phi^{a}\right|_{\kappa(c)} ^{c}, \quad \text { for } c \in \mathcal{A}_{h}^{\mathrm{nd}}(a)
\end{aligned}
$$

and $i_{h}\left(\nabla \phi^{a}\right)^{c}=0$ otherwise. It proves the theorem.

The inequality $r_{p} \leqslant r_{u}+1$ will naturally be satisfied. The cases of interest are $r_{u}=r_{p}$ (for implementation simplicity) or $r_{u}=r_{p}+1$ (from a priori estimates, see Corollary 9). Otherwise, we should simply use formula (5).

In terms of implementation, it is better to build the stabilization terms as indicated in Algorithm 1 (for the most usual elementbased integration/assembly); the equivalence is easily checked. Algorithm 1 implements the stabilization matrix for method (6). We can still implement this algorithm in different ways, depending on the treatment of $\left.\nabla \phi^{a}\right|_{\kappa(c)} ^{c}$. We can consider to store this array, which has the size of the velocity unknowns array times the number of nodes per element, i.e. for linear simplicial FEs this factor is 
$d+1$. This memory usage is acceptable in an implicit FE code compared to preconditioned solver requirements and it is well-suited for parallelization. Otherwise, it can be computed every time is used, with a slight growth of the operation counter. Finally let us note that we are not required to store it for interior nodes, and that vertex-based and edge-based integration algorithms could be considered instead.

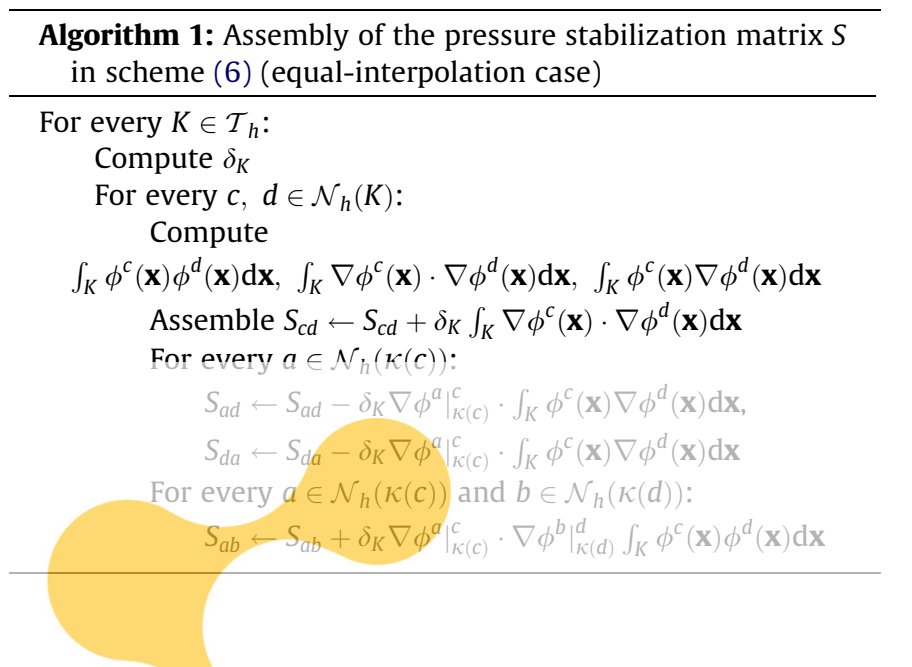

Remark 3. In principle, we could consider the Clément interpolant [10] as on altemative to the Scott-Zhan interpolation is still local. However, the a projection and does not satisfy homo tions, in case it is required. The fact tha some undesired effects, e.g. the resultin pateh test. That is to say, for an exac solution $u_{h}$ differs from $u$ in general. Further, let us show in Fig. 1 the stencil for a FE, in the worst-case and best-case scenario, as

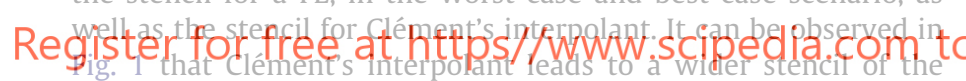
resulting matrix (a node is coupled to its neighbors' neighbors). These facts motivated the introduction of the Scott-Zhang-type projector (5).

\section{Numerical analysis}

In this section, we analyze the stability and accuracy of the numerical method (6) for both (7) and (8) versions of the stabilization term. Let $h_{K}$ denote the diameter of $K \in \mathcal{T}_{h}$ and $h:=\max _{K \in \mathcal{T}_{h}} h_{K}$. We assume a non-degenerate partition: there exists a constant $\sigma \in(1, \infty)$ independent of $h$ such that $\max _{K \in \mathcal{T}_{h}} \frac{h_{K}}{\rho_{K}} \leqslant \sigma$, where $\rho_{K}$ denotes the radius of the largest closed ball contained in $\bar{K}$. The analysis is simpler for regular partitions, i.e. $\min _{K \in \mathcal{T}_{h}} \rho_{K} \geqslant \sigma h$. We will make use of the local inverse estimate

$\left\|\nabla v_{h}\right\|_{K} \leqslant c_{i} h_{K}^{-1}\left\|v_{h}\right\|_{K}, \quad$ for any $K \in \mathcal{T}_{h}$,

where $c_{i}>0$ can depend on $\sigma$ (as other constants defined below) but not on $h$. Further, we use the general approximation theory of the Scott-Zhang projector. Let $v \in W_{p}^{k}(\Omega)$ and $0 \leqslant s \leqslant k \leqslant r+1$ : there exists $c_{e}>0$ independent of $h$ such that

$$
\begin{aligned}
\left\|v-i_{h}(v)\right\|_{W_{p}^{s}(K)} & \leqslant c_{e} h_{K}^{k-s}\|v\|_{W_{p}^{k}\left(S_{K}\right)}, \quad\left(\sum_{K \in \mathcal{T}_{h}}\left\|v-i_{h}(v)\right\|_{W_{p}^{s}(K)}^{p}\right)^{\frac{1}{p}} \\
& \leqslant c_{e} h^{k-s}\|v\|_{W_{p}^{k}(\Omega)},
\end{aligned}
$$

where $S_{K}$ is a domain made of the elements neighboring $K$ (see [6]) and $r$ denotes the order of approximation of the corresponding FE space. The global estimate holds for non-degenerate partitions only. The same estimates hold for $i_{h, 0}$ (the interpolant that keeps null traces) as soon as $v \in H_{0}^{1}(\Omega)$.

Finally, the symbol $\lesssim$ is used to denote $<$ up to a positive constant independent of $h$ (idem for $\gtrsim$ and $\approx$ ).

3.1. Stability results

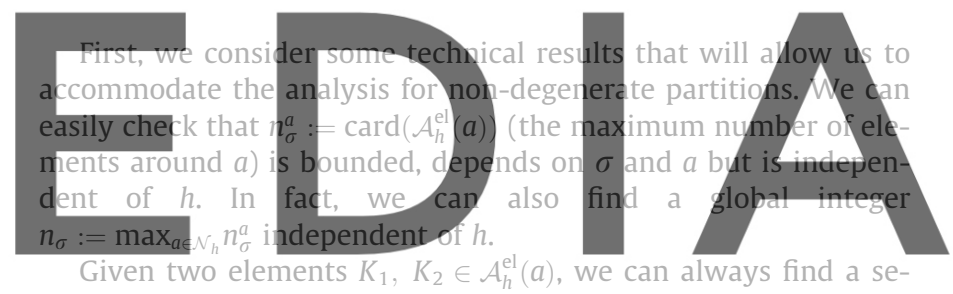
quence of elements

\section{downlgad the versjion whithput the watermark \\ where $m \leqslant n^{a}$.}

such that $K_{12}^{i}$ and $K_{12}^{i+1}(i=1, \ldots, m-1)$ share an edge (for $d=2,3$ ). We have the following lemma.

Lemma 4. Given a non-degenerate partition $\mathcal{T}_{h}$, for every node $a \in \mathcal{N}_{h}$, there exists $h_{a}>0$ such that:

$\sigma^{-\tilde{n}_{\sigma}^{a}+1} h_{K} \leqslant h_{a} \leqslant \sigma^{\tilde{n}_{\sigma}^{a}-1} h_{K}, \quad$ for any $K \in \mathcal{A}_{h}^{\mathrm{el}}(a)$,

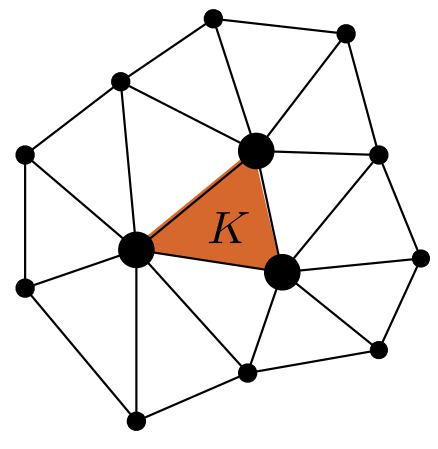

(a)

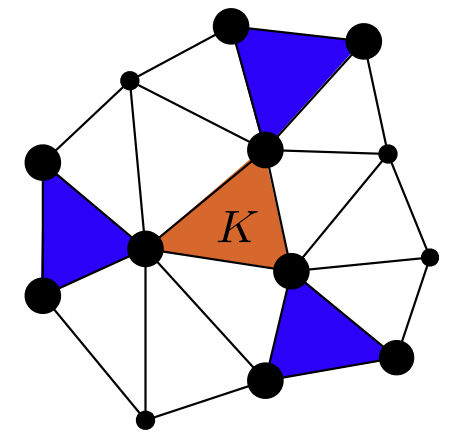

(b)

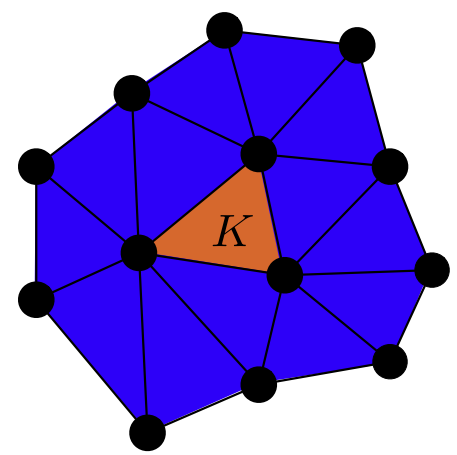

(c)

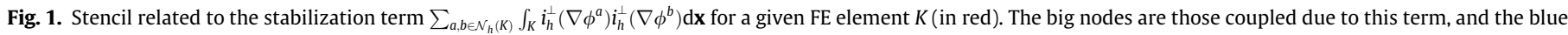

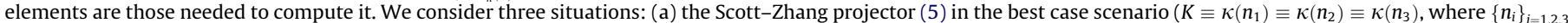

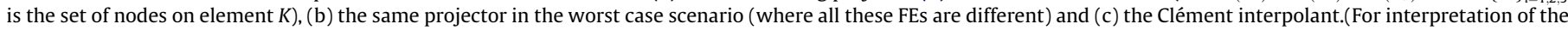
references to colour in this figure legend, the reader is referred to the web version of this article.) 
where

$\tilde{n}_{\sigma}^{a}\left(K_{i}\right):=\max _{K_{j} \in \mathcal{A}_{h}^{\mathrm{el}}(a)} \operatorname{card}\left(\mathcal{P}_{h}\left(a, K_{i}, K_{j}\right)\right), \quad \tilde{n}_{\sigma}^{a}:=\min _{K_{i} \in \mathcal{A}_{h}^{\mathrm{le}}(a)} \tilde{n}_{\sigma}^{a}\left(K_{i}\right) \leqslant n_{\sigma}^{a}$.

Proof. Suppose the affine mapping $F_{K}(\tilde{\mathbf{x}})=B \tilde{\mathbf{x}}+\mathbf{x}_{0}$. Given two elements $K_{1}, K_{2}$ sharing an edge, denoted by $E$, we can easily prove (see [9, Th. 3.1.3]):

$\left|B_{K_{i}}^{-1}\right|^{-1} \leqslant \operatorname{meas}(E) \leqslant\left|B_{K_{i}}\right|, \quad\left|B_{K_{i}}\right| \leqslant h_{K_{i}}, \quad\left|B_{K_{i}}^{-1}\right| \leqslant \rho_{K_{i}}^{-1}, \quad$ for $i=1,2$.

Therefore, we get $\sigma^{-1} h_{K_{1}} \leqslant h_{K_{2}} \leqslant \sigma h_{K_{1}}$. Now, let us take $K_{1} \in \arg \min \tilde{n}_{\sigma}^{a}\left(K_{i}\right)$ and define $h^{a}:=h_{K_{1}}$. For any $K_{j} \in \mathcal{A}_{h}^{\mathrm{el}}(a)$, using $K_{i} \in \mathcal{A}_{h}^{\mathrm{el}}(a)$

the definition of the sequence $\mathcal{P}_{h}\left(a, K_{1}, K_{j}\right)$, we have:

$\max _{K_{j} \in \mathcal{A}_{h_{h}^{\mathrm{e}}(a)}} \frac{h_{a}}{h_{K_{j}}}=\max _{K_{j} \in \mathcal{A}_{h}^{\mathrm{el}}(a)} \frac{h_{K_{1}}}{h_{K_{1 j}^{2}}} \ldots \frac{h_{K_{1 j}^{m-1}}}{h_{K_{j}}} \leqslant \sigma^{\tilde{n}_{\sigma}^{a}-1}$.

By its definition, $\tilde{n}_{\sigma}^{a} \leqslant n_{\sigma}^{a}$. Let us note that for nodes on faces $n_{\sigma}^{a}=2$ and on interior nodes the same result applies for $n_{\sigma}^{a}=1$. It proves the lemma.

Let us define the $\delta$-weighted norm $\|v\|_{\delta}:=(v, v)_{\delta}^{\frac{1}{2}}$, with the definition of the $\delta$-weighted inner product in (7) or (8), depending on the case considered. Finally, we note that $\nabla Q_{h} \subset D_{h}$, and so $\delta\left(i_{h}^{\perp}\left(\nabla p_{h}\right)\right)$ is well-defined. In the next theorem, we state the stabil-

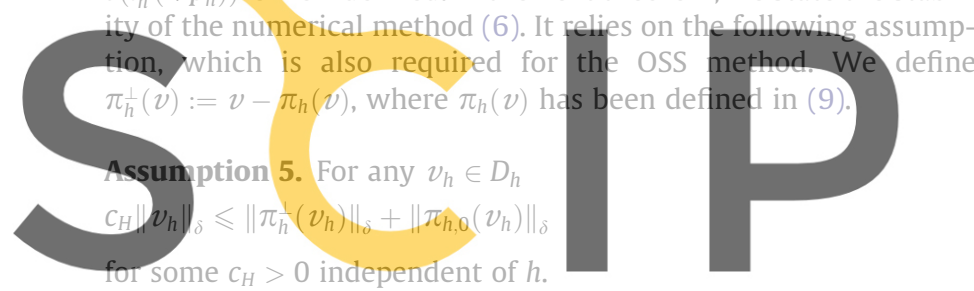

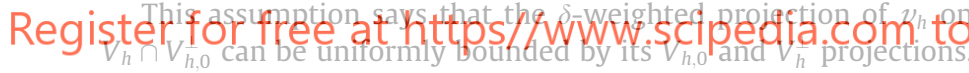
We refer to [12] for the proof that this condition holds for simplicial meshes. The OSS formulation also relies on this assumption, as soon as we consider the projector $\pi_{h}(\cdot)$. Both OSS with $\pi_{h, 0}(\cdot)$ and NPS with $i_{h, 0}(\cdot)$ do not need this assumption anymore.

Theorem 6. For the definition of the $\delta$-product in (7) or (8), for any $p_{h} \in Q_{h}$

$\beta\left\|p_{h}\right\|_{Q} \leqslant \sup _{v_{h} \in V_{h, 0}} \frac{\left(p_{h}, \nabla \cdot v_{h}\right)}{\left\|v_{h}\right\|_{V}}+\left\|i_{h}^{\perp}\left(\nabla p_{h}\right)\right\|_{\delta}$

for some $\beta>0$ independent of $h$.

Proof. Let us consider the numerical analysis for the element-wise case (7), since the one for the nodal-based case (8) is slightly simpler. Since $\delta\left(\pi_{h, 0}\left(\nabla p_{h}\right)\right) \in V_{h, 0}$ by construction, we get:

$$
\begin{aligned}
-\left(p_{h}, \nabla \cdot \delta\left(\pi_{h, 0}\left(\nabla p_{h}\right)\right)\right) & =\left(\nabla p_{h}, \delta\left(\pi_{h, 0}\left(\nabla p_{h}\right)\right)\right) \\
& =\left(\pi_{h, 0}\left(\nabla p_{h}\right), \delta\left(\pi_{h, 0}\left(\nabla p_{h}\right)\right)\right) \\
& =\sum_{K \in \mathcal{T}_{h} a, b \in \mathcal{N}_{h}(K)} \delta_{a} \pi_{h, 0}\left(\nabla p_{h}\right)^{a} \pi_{h, 0}\left(\nabla p_{h}\right)^{b}\left(\phi^{a}, \phi^{b}\right)_{K} \\
& \gtrsim\left\|\pi_{h, 0}\left(\nabla p_{h}\right)\right\|_{\delta} .
\end{aligned}
$$

We have used the definition of the projector (10) and the inequality $c_{1}^{-1} \delta_{K} \leqslant \delta_{a} \leqslant c_{1} \delta_{K}$ for $0<c_{1} \leqslant \sigma^{2\left(n_{\sigma}-1\right)}$, consequence of Lemma 4 . Using the local inverse inequality (12), we get

$$
\left.\left\|\delta\left(\pi_{h, 0}\left(\nabla p_{h}\right)\right)\right\|_{V}^{2} \leqslant \sum_{K \in \mathcal{T}_{h}} v h_{K}^{-2} \| \delta\left(\pi_{h, 0}\left(\nabla p_{h}\right)\right)\right)\left\|_{K}^{2} \lesssim\right\| \pi_{h, 0}\left(\nabla p_{h}\right) \|_{\delta} .
$$

Combining these results, we get:

$\left\|\pi_{h, 0}\left(\nabla p_{h}\right)\right\|_{\delta} \lesssim \sup _{v_{h} \in V_{h, 0}} \frac{\left(p_{h}, \nabla \cdot v_{h}\right)}{\left\|v_{h}\right\|_{V}}$

Clearly, the stabilization term provides control over $\left(i_{h}^{\perp}\left(\nabla p_{h}\right)\right.$, $\left.i_{h}^{\perp}\left(\nabla p_{h}\right)\right)_{\delta}=\left\|i_{h}^{\perp}\left(\nabla p_{h}\right)\right\|_{\delta}^{2}$. Let us recall the following result, that follows from [14, Lemma 9.7] and the fact that $\left(\phi^{a}, \phi^{a}\right)_{K}=h_{K}^{d}$ for any $a \in \mathcal{N}_{h}(K)$. For any FE $K \in \mathcal{T}_{h}$ and any FE function $v_{h} \in D_{h}$

$$
\begin{aligned}
c_{m}^{-1} \sum_{a \in \mathcal{N}_{h}(K)}\left(v_{h}^{a}\right)^{2}\left(\phi^{a}, \phi^{a}\right)_{K} & \leqslant \sum_{a, b \in \mathcal{N}_{h}(K)} v_{h}^{a} v_{h}^{b}\left(\phi^{a}, \phi^{b}\right)_{K} \\
& \leqslant c_{m} \sum_{a \in \mathcal{N}_{h}(K)}\left(v_{h}^{a}\right)^{2}\left(\phi^{a}, \phi^{a}\right)_{K}
\end{aligned}
$$

for a constant $c_{m}>0$ that does not depend on $\sigma$ or $h$. We have:

$$
\begin{aligned}
\left\|\pi_{h}^{\perp}\left(\nabla p_{h}\right)\right\|_{\delta}^{2} & \lesssim \sum_{K \in \mathcal{T}_{h} a \in \mathcal{N}_{h}(K)} \delta_{K}\left(\pi_{h}^{\perp}\left(\nabla p_{h}\right)^{a}\right)^{2}\left(\phi^{a}, \phi^{a}\right)_{K} \lesssim\left(\pi_{h}^{\perp}\left(\nabla p_{h}\right), \delta\left(\pi_{h}^{\perp} \nabla p_{h}\right)\right) \\
& =\left(\pi_{h}^{\perp}\left(\nabla p_{h}\right), \delta\left(\pi_{h}^{\perp}\left(\nabla p_{h}\right)\right)+\left(\pi_{h}^{\perp}\left(\nabla p_{h}\right), \delta\left(\pi_{h}\left(\nabla p_{h}\right)-i_{h}\left(\nabla p_{h}\right)\right)\right.\right. \\
& =\left(\pi_{h}^{\perp}\left(\nabla p_{h}\right), \delta\left(i_{h}^{\perp}\left(\nabla p_{h}\right)\right)\right. \\
& \lesssim\left\|\pi_{h}^{\perp}\left(\nabla p_{h}\right)\right\|_{\delta}\left\|i_{h}^{\perp}\left(\nabla p_{h}\right)\right\|_{\delta},
\end{aligned}
$$

where we have used the relation $\left(\pi_{h}^{\perp}\left(\nabla p_{h}\right), w_{h}\right)=0$ for any $w_{h} \in V_{h}$, obtained from (9). Combining these results with Assumption 5 we get:

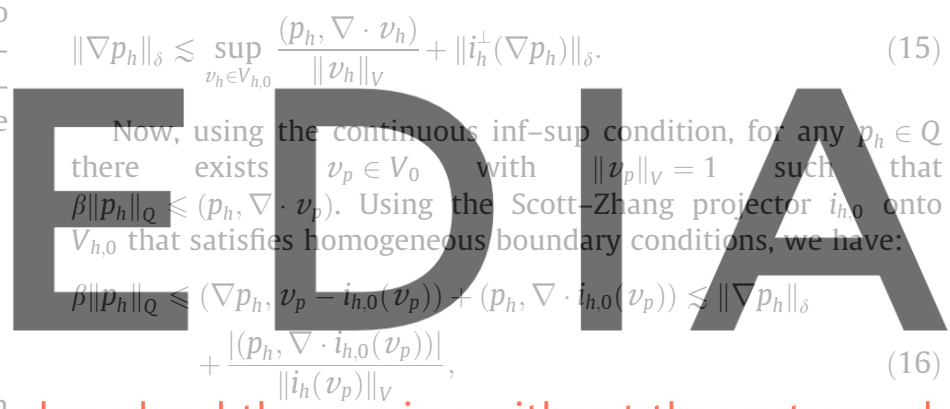

download the version without the watermark

(13) and the stability of the Scott-Zhang projector (obtained from (13) for $k=s$ ). We prove the theorem invoking (15) in (16). The analysis for the nodal definition of $\delta$ in (8) follows in a similar way, with slight modifications. It is based on the fact that $\left(\delta^{\frac{1}{2}}\left(v_{h}\right), \delta^{\frac{1}{2}}\left(v_{h}\right)\right) \approx\left(v_{h}, \delta\left(v_{h}\right)\right)$, which is a consequence of $(14)$.

The analysis for $i_{h, 0}(\cdot)$ is straightforward and does not require Assumption 5. The following corollary is a direct consequence of this theorem and the coercivity of the viscous term $v(\nabla u, \nabla u)$ $=\|u\|_{V}^{2}$.

Corollary 7. The stabilized bilinear form $c_{h}(\cdot)$ defined in (6) and (7) (or (6)-(8)) satisfies

$\beta \leqslant \inf _{\left(u_{h}, p_{h}\right) \in V_{h, 0} \times Q_{h}} \sup _{\left(v_{h}, q_{h}\right) \in V_{h, 0} \times Q_{h}} \frac{c_{h}\left(u_{h}, p_{h}, v_{h}, q_{h}\right)}{\left(\left\|u_{h}\right\|_{V}+\left\|p_{h}\right\|_{Q}\right)\left(\left\|v_{h}\right\|_{V}+\left\|p_{h}\right\|_{Q}\right)}$

for some $\beta>0$ independent of $h$.

\subsection{Convergence analysis}

Finally, we show that method (6) is optimally convergent.

Theorem 8. The solution $\left(u_{h}, p_{h}\right)$ of the discrete problem (6) and (7) (or (6)-(8)) satisfies:

$$
\begin{gathered}
\left\|u-u_{h}\right\|_{V}+\left\|p-p_{h}\right\| \lesssim \inf _{\left(w_{h}, r_{h}\right) \in V_{h, 0} \times Q_{h}}\left(\left\|u-w_{h}\right\|_{V}+\left\|p-r_{h}\right\|_{Q}\right) \\
+\left\|i_{h}^{\perp}(\nabla p)\right\|_{\delta} .
\end{gathered}
$$




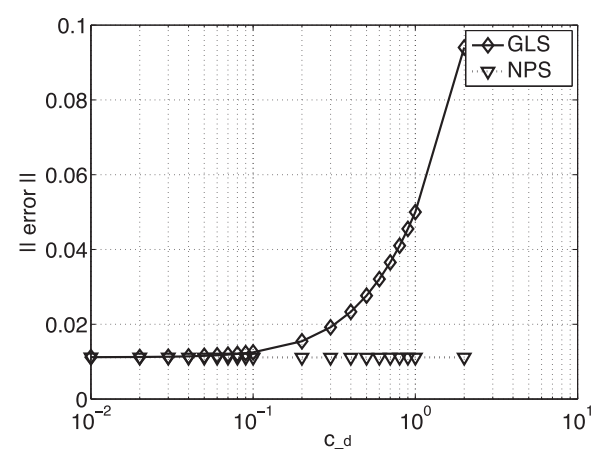

(a) $\left\|\boldsymbol{u}_{h}-\boldsymbol{u}\right\|$

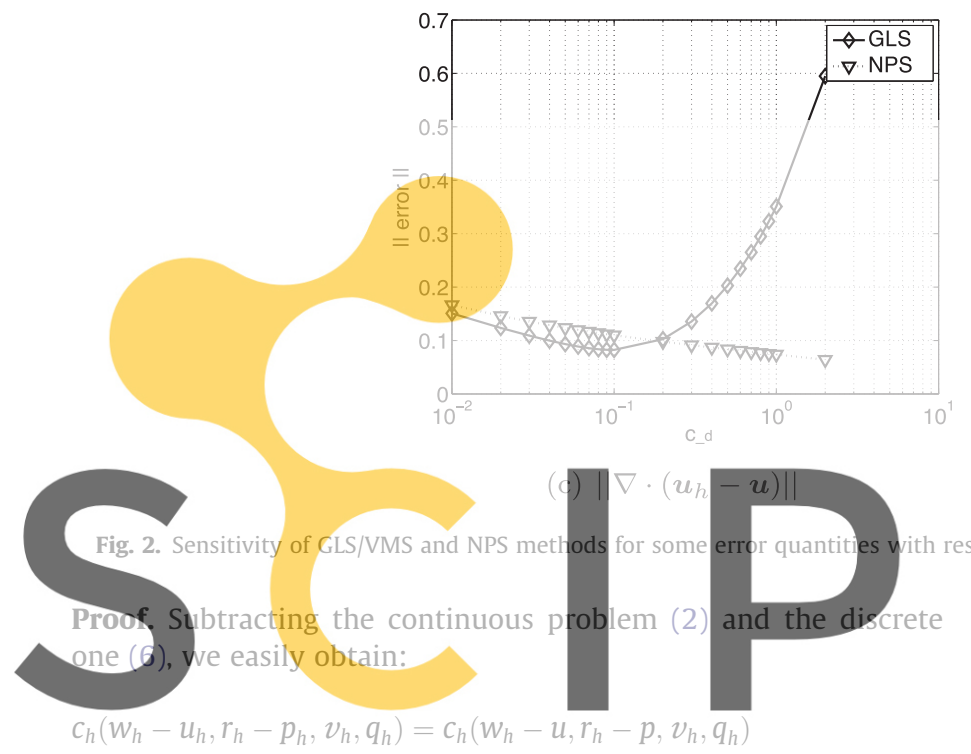

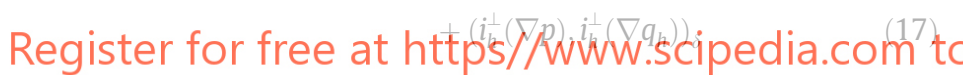
for any $\left(w_{h}, r_{h}\right),\left(v_{h}, q_{h}\right) \in V_{h, 0} \times Q_{h}$. Using the local estimate in (13) with $k=l$ and the local inverse inequality (12), we have

$\left\|i_{h}^{\perp}\left(\nabla r_{h}\right)\right\|_{\delta}^{2} \lesssim \sum_{K \in \mathcal{T}_{h}} \delta_{K}\left\|\nabla r_{h}\right\|_{S_{K}}^{2} \lesssim \sum_{K \in \mathcal{T}_{h}} \delta_{K} h_{K}^{-2}\left\|r_{h}\right\|_{S_{K}}^{2} \lesssim\left\|r_{h}\right\|_{Q}^{2}$

This result can be used to prove the continuity of the bilinear form $c_{h}\left(w_{h}-u_{h}, r_{h}-p_{h}, v_{h}, q_{h}\right)$ in $V \times Q$, i.e.

$$
\begin{aligned}
c_{h}\left(w_{h}, r_{h}, v_{h}, q_{h}\right) \leqslant & \left(\left\|w_{h}\right\|_{V}+\left\|r_{h}\right\|_{Q}\right) \times\left(\left\|v_{h}\right\|_{V}+\left\|q_{h}\right\|_{Q}\right), \\
& \text { for any }\left(w_{h}, r_{h}\right),\left(v_{h}, q_{h}\right) \in V_{h, 0} \times Q_{h} .
\end{aligned}
$$

Finally, invoking the stability result in Corollary 7 and inequalities (18) and (19) in (17), we obtain:

$\left\|w_{h}-u_{h}\right\|_{V}+\left\|r_{h}-p_{h}\right\|_{Q} \lesssim\left\|u-w_{h}\right\|_{V}+\left\|p-r_{h}\right\|_{Q}+\left\|i_{h}^{\perp}(\nabla p)\right\|_{\delta}$.

Using the triangle inequality we prove the theorem.

As a consequence, using e.g. the approximability properties of the Scott-Zhang projector, we have the following corollary.

Corollary 9. Let us assume that the solution of the continuous problem (1) satisfies $(u, p) \in H^{1+\alpha}(\Omega) \times H^{\alpha}(\Omega)$ for some $\alpha \geqslant 1$. The solution $\left(u_{h}, p_{h}\right)$ of the discrete problem (6) and (7) (or (6)-(8)) satisfies:

$$
\begin{gathered}
\left\|u-u_{h}\right\|_{V}+\left\|p-p_{h}\right\| \lesssim h^{\mu}\left(v^{\frac{1}{2}}\|u\|_{H^{1+\mu}(\Omega)}+v^{-\frac{1}{2}}\left\|p-r_{h}\right\|_{H^{\mu}(\Omega)}\right), \\
\text { with } \mu:=\min \left(r_{u}, r_{p}+1, \alpha\right),
\end{gathered}
$$

where $r_{u}$ and $r_{p}$ are the order of approximation of $V_{h}$ and $Q_{h}$ respectively.

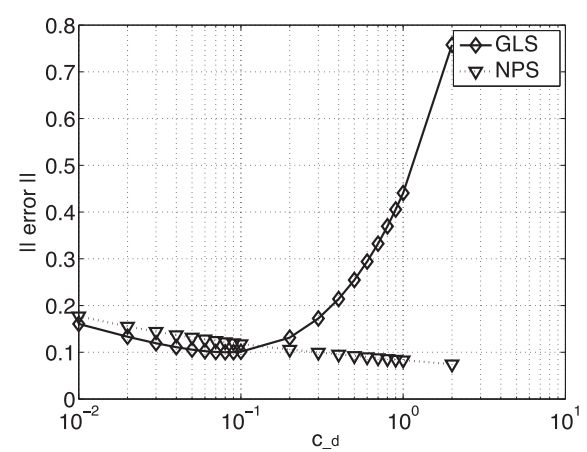

(b) $\left\|\nabla\left(\boldsymbol{u}_{h}-\boldsymbol{u}\right)\right\|$
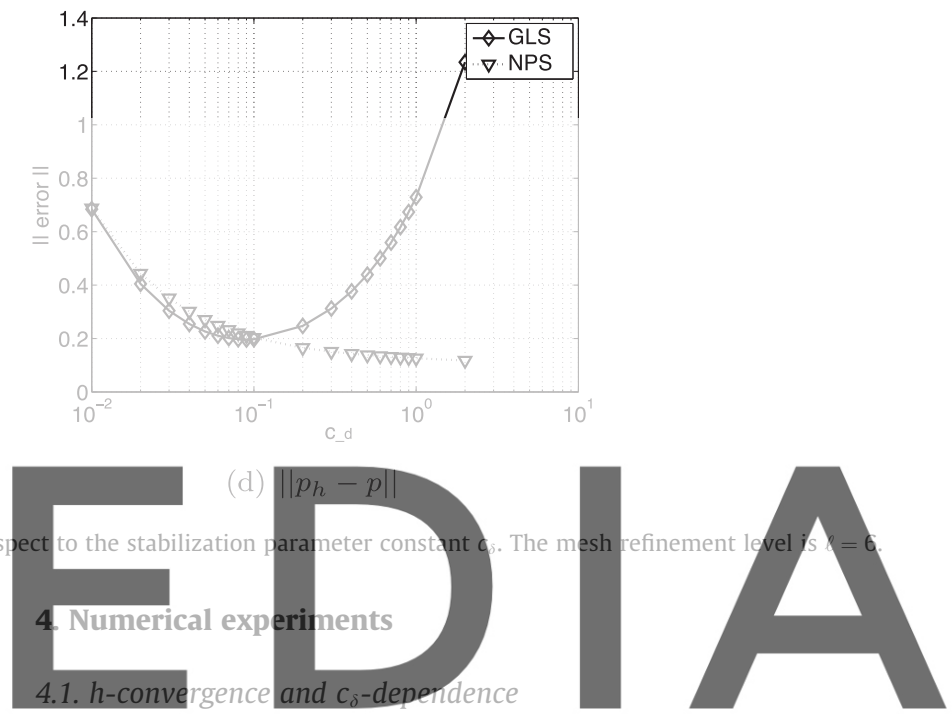

In order to test the convergence of the proposed method, we

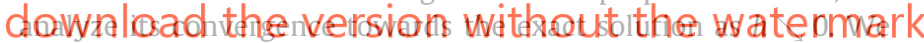
have considered the Stokes problem on the unit square with exact solution:

$u(x, y)=\left(20 x y^{3}, 5 x^{4}-5 y^{4}\right), \quad p(x, y)=60 x^{2} y-20 y^{3}$.

This test problem has been proposed in [20] and also used in [5]. We have compared the GLS method, which coincides with VMS for linear FEs, and the NPS method in (6) and (7). We have used a family of regular triangular FE meshes with $h=2^{-l}$, with $\ell=1,2,3,4,5,6$. For this test problems, the difference between choices (7) and (8) is not appreciable, since the family of meshes used in the convergence analysis are regular. The difference between the element-wise and nodal-wise definition of the stabilization parameter is expected to be more significant for non-degenerate meshes, i.e. using adaptive refinement, and remarkable for anisotropic meshes.

First of all, we have analyzed the sensitivity of both the GLS formulation (3) and the NPS formulation (6). In Fig. 2 we have plotted some quantities of the velocity and pressure error with respect to $c_{\delta}$. The GLS method has an optimal behavior at $c_{\delta} \sim 0.1$ for all quantities, but increasing $c_{\delta}$ has a clear negative impact on accuracy. On the contrary, the NPS formulation is quite insensitive to $c_{\delta}$; this behavior is particularly relevant for the $L^{2}$-norm of the velocity error in Fig. 2(a). Further, the NPS formulation seems to sligthly improve for larger values of $c_{\delta}$.

Taking the optimal value $c_{\delta}=0.1$ for the GLS formulation, we have plotted the error against the mesh size $h$ in Fig. 3 for both methods. We observe an almost identical behavior for both formulations. Optimal quadratic convergence is attained for the $L^{2}$-norm of the velocity error, and a super-convergence order of $\frac{3}{2}$ is observed for the rest of quantities. 


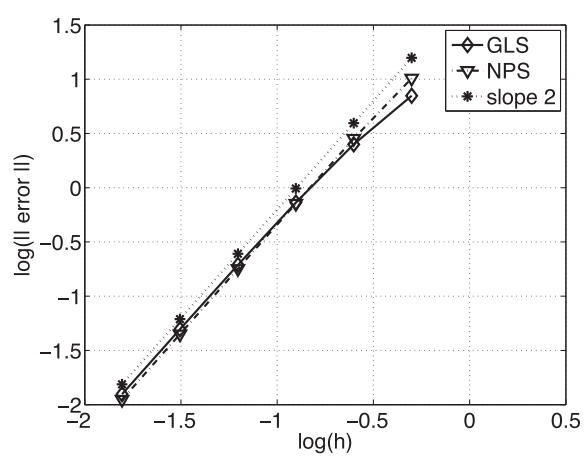

(a) $\left\|\boldsymbol{u}_{h}-\boldsymbol{u}\right\|$
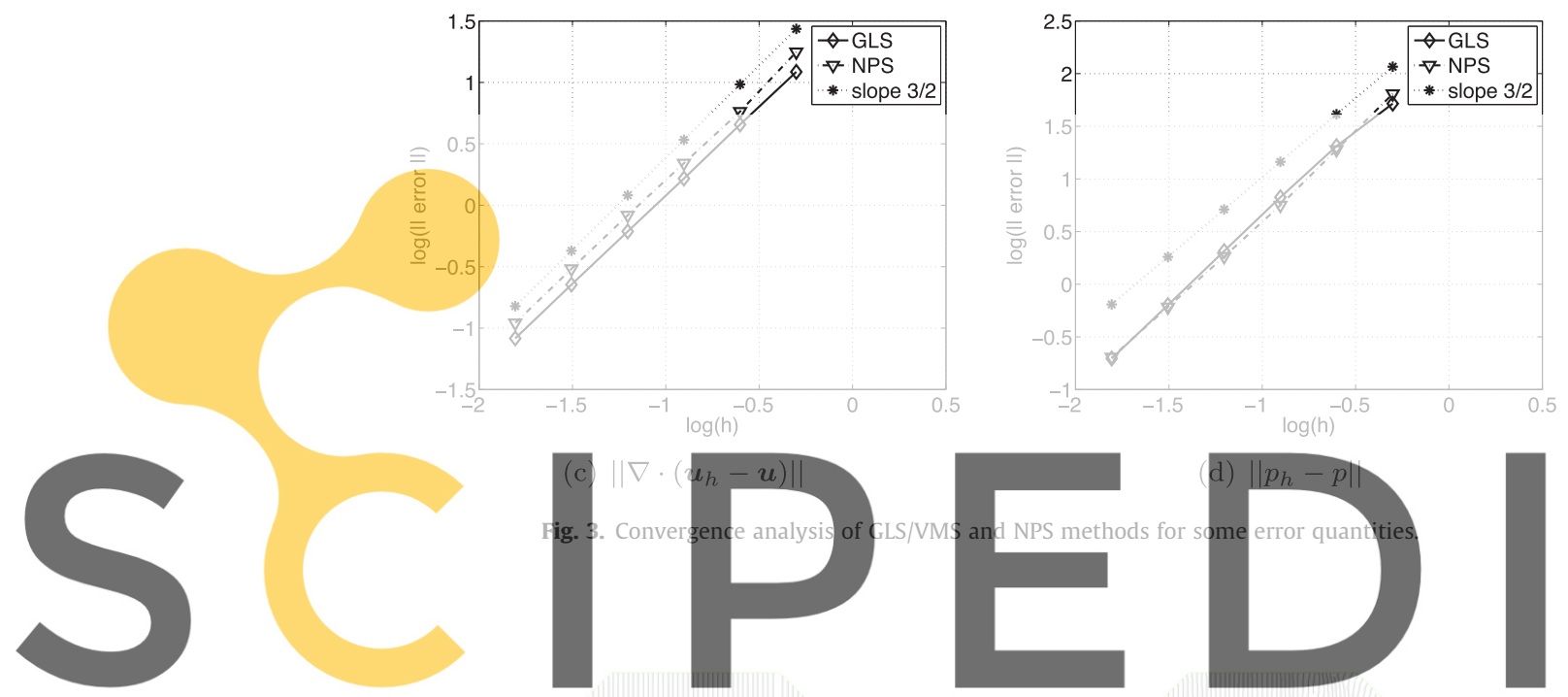

(b) $\left\|\nabla\left(\boldsymbol{u}_{h}-\boldsymbol{u}\right)\right\|$
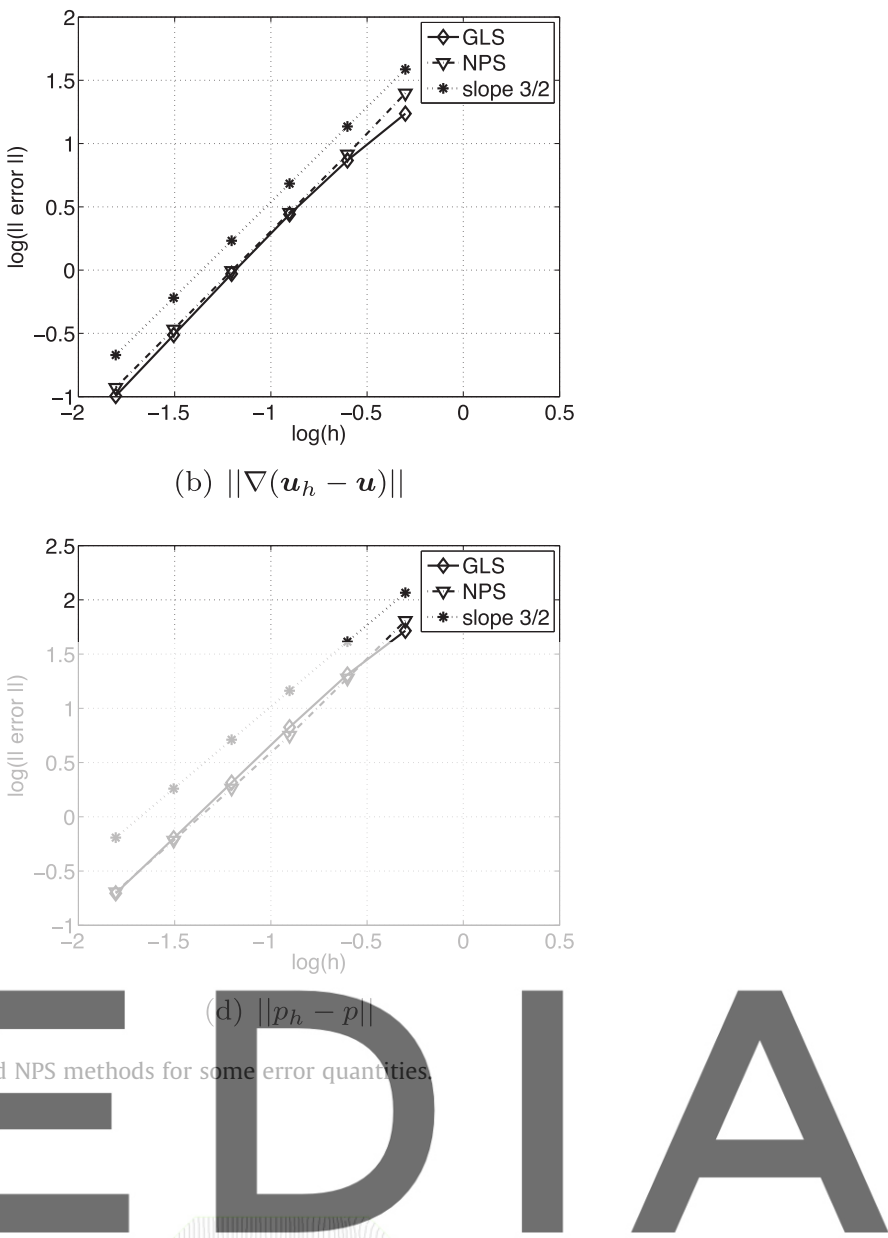

Register for free at https//www.scipedia.com to download the version without the watermark

(a) NPS formulation

(b) GLS/VMS formulation

Fig. 4. Contour lines of the pressure field for the Poiseuille flow test.

\subsection{Pressure error on the boundary}

The artificial pressure boundary layers that appear with GLS/ VMS formulations are well-known. Let us consider the Poiseuille flow test in [12] for a structured mesh of 1,250 linear triangular FEs; we refer to this work for a complete discussion about the misbehavior of the GLS/VMS formulation. We show the pressure contours for both formulations in Fig. 4. The GLS/VMS formulation produces a wrong solution, motivated by the fact that $\partial_{n} p=0$ is weakly enforced on the boundary. On the other hand, even though $u \notin V_{h}$, the NPS solution leads to the exact pressure, as the LPS formulation in [5] (see this reference for a super-convergence proof).

\section{Conclusions}

In this work, we have considered a symmetric stabilization method based on a non-standard version of the Scott-Zhang projector that is well-posed for $L^{1}(\Omega)$ functions. This low regularity requirement is needed, in order to project FE pressure gradients, which are discontinuous element-wise polynomials. Further, the application of this projector over this type of functions is very simple and cheap and allows for an efficient implementation of the method.
This projector has important benefits compared to the existing choices: the $L^{2}(\Omega)$ projector in OSS [12] and the local projections in LPS techniques $[5,19]$. On one hand, the projector is local, i.e. no global linear system must be solved to compute the projections. It allows to explicitly have the system matrix in memory, which is required e.g. for ILU preconditioning of the full FE system matrix, the use of direct solvers or substructuring domain decomposition techniques for parallelization. Further, for iterative solvers, a global projector requires nested solvers, one for the FE problem and another one (per iteration) for the projection computation. So, the local projection is very desirable in order to facilitate implementation, reduce CPU cost and work on distributed memory machines. In particular, the extended stencil of the proposed method seems to be minimal. On the other hand, the proposed method lightens the requirements of LPS techniques, since it does not require any particular macro-element topology of the mesh or bubble-enriched FE spaces.

Different alternatives of the method have been proposed, depending on the nature of the stabilization parameter (nodal vs. element definition) and the enforcement of Dirichlet boundary conditions in the projector. Some implementation issues have been addressed. Numerical analysis shows optimal stability and convergence properties, which have also been checked via numerical 
experimentation. Compared to the GLS/VMS formulations, the resulting method is less sensitive to the choice of the stabilization parameter and does not exhibit the pressure misbehavior on the boundary.

The method has been presented for the Stokes problem. However, we can apply it to Darcy's or Maxwell's systems, using the ideas in [2], by replacing the $L^{2}(\Omega)$ projections by the Scott-Zhang projector proposed herein.

\section{Acknowledgements}

This work has been funded by the European Research Council under the FP7 Programme Ideas through the Starting Grant No. 258443 - COMFUS: Computational Methods for Fusion Technology.

\section{References}

[1] S. Badia, R. Codina, Unified stabilized finite element formulations for the Stokes and the Darcy problems, SIAM Journal on Numerical Analysis 47 (3) (2009) 1971

[2] S. Badia, R. Codina, Stokes, Maxwell and Darcy: a single finite element approximation for three model problems, Applied Numerical Mathematics 62 (4) (2012) 246-263.

[3] S. Badia, R. Codina, R. Planas, On an unconditionally convergent stabilized finite element approximation of resistive magnetohydrodynamics, UPcommons (2011). http://www.hdl.handle.net/2117/15755.

[4] S. Badia, R. Planas, J.V. Gutiérrez-Santacreu, Unconditionally stable operator splitting algorithms for the incompressible magnetohydrodynamics (MHD) system discretized by a stabilized finite element formulation based on

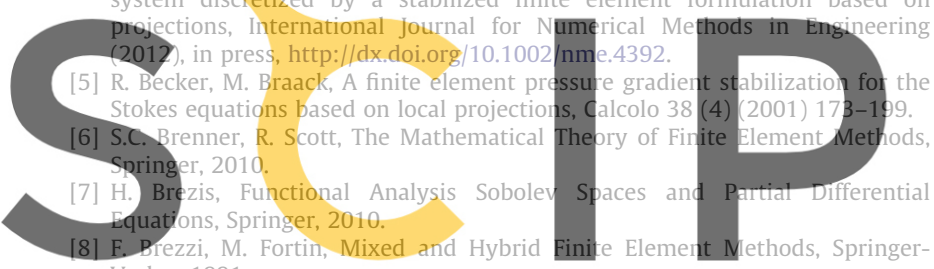
Verlag, 1991

[9] P.G. Ciarlet, The Finite Element Method for Elliptic Problems, second ed., SIAM: Regisociety for Industrial and Applied Mathematics, 2002.
[11] R. Codina, Stabilized finite element approximation of transient incompressible flows using orthogonal subscales, Computer Methods in Applied Mechanics and Engineering 191 (39-40) (2002) 4295-4321.

[12] R. Codina, J. Blasco, A finite element formulation for the Stokes problem allowing equal velocity-pressure interpolation, Computer Methods in Applied Mechanics and Engineering 143 (3-4) (1997) 373-391.

[13] R. Codina, Javier Principe, Oriol Guasch, S. Badia, Time dependent subscales in the stabilized finite element approximation of incompressible flow problems, Computer Methods in Applied Mechanics and Engineering 196 (21-24) (2007) 2413-2430.

[14] A. Ern, J.-L. Guermond, Theory and Practice of Finite Elements, Springer, 2004

[15] V. Girault, J-L. Lions, Two-grid finite-element schemes for the transient Navier-Stokes problem, ESAIM: Mathematical Modelling and Numerical Analysis 35 (5) (2002) 945-980.

[16] T.J.R. Hughes, L.P. Franca, M. Balestra, A new finite element formulation for computational fluid dynamics: V. Circumventing the Babuska-Brezzi condition: a stable Petrov-Galerkin formulation of the stokes problem accommodating equal-order interpolations, Computer Methods in Applied Mechanics and Engineering 59 (1) (1986) 85-99.

[17] T.J.R. Hughes, Multiscale phenomena: Green's functions, the Dirichlet-toNeumann formulation, subgrid scale models, bubbles and the origins of stabilized methods, Computer Methods in Applied Mechanics and Engineering 127 (1-4) (1995) 387-401.

[18] K.E. Jansen, S.S. Collis, C. Whiting, F. Shakib, A better consistency for low-order stabilized finite element methods, Computer Methods in Applied Mechanics and Engineering 174 (1-2) (1999) 153-170.

[19] G. Matthies, P. Skrzypacz, L. Tobiska, A unified convergence analysis for local projection stabilisations applied to the Oseen problem, ESAIM: Mathematical Modelling and Numerical Analysis 41 (4) (2007) 713-742.

[20] S. Norburn, D. Silvester, Stabilised vs. stable mixed methods for incompressible flow, Computer Methods in Applied Mechanics and Engineering 166 (1-2) (1998) 131-141.

[21] R. Planas, S. Badia, R. Codina, Approximation of the inductionless MHD problem using a stabilized finite element method, Journal of Computational Physics 230 (8) (2011) 2977-2996

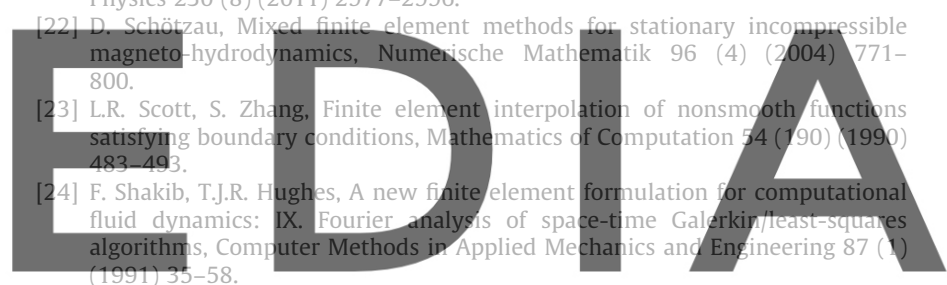

[25] A. Toselli, O. Widlund, Domain Decomposition Methods, first ed., Springer

download the version without the watermark

regularization, ESAIM: Mathematical Modelling and Numerical Analysis 9(R2) (1975). 